PACS NUMBER: 42.50.-P, 72.20.JV;

UDK 539.184

\title{
ENERGY APPROACH TO ELECTRON CAPTURE AND IONIZATION PROCESSES IN ION-ATOMIC COLLISION SYSTEM
}

\author{
A. V. Loboda \\ Odessa State Environmental University, Odessa, Ukraine \\ ENERGY APPROACH TO ELECTRON CAPTURE AND IONIZATION PROCESSES \\ IN ION-ATOMIC COLLISION SYSTEM
}

\begin{abstract}
A. V. Loboda
Abstract. Energy approach is generalized to calculate the electron capture cross sections in the $\mathrm{H}^{+}+$ $\mathrm{H}(1 \mathrm{~s})$ collision system. The numerical results are presented for collision energies 10 and $100 \mathrm{keV}$

Keywords: ion-atomic collision system, energy approach

\section{ЭНЕРГЕТИЧЕСКИЙ ПОДХОД К ИЗУЧЕНИЮ ПРОЦЕССОВ ЗАХВАТА ЭЛЕКТРОНА И ИОНИЗАЦИИ В ИОН-АТОМНОЙ СТОЛКНОВИТЕЛЬНОЙ СИСТЕМЕ}

\section{А. В. Лобода}

Аннотация. Энергетический подход обобщен с целью расчета сечения захвата электрона в ион-атомной столкновительной системе $\mathrm{H}^{+}+\mathrm{H}(1 \mathrm{~s})$. Численные оценки получены для энергий столкновения 10 и 100 кэВ.
\end{abstract}

Ключевые слова: ион-атомная столкновительная система, энергетический подход

\section{ЕНЕРГЕТИЧНІЙ ПІДХІД ДО ВИВЧЕННЯ ПРОЦЕСІВ ЗАХОПЛЕННЯ ЕЛЕКТРОНУ ТА ІОНІЗАЦІЇ В ІОН-АТОМНІЙ СИСТЕМІ ІЗ ЗІТКНЕННЯМ}

\section{А. В. Лобода}

Анотація. Енергетичний підхід узагальнено з метою розрахунку перерізу захоплення електрону в іон-атомній системі у стані зіткнення $\mathrm{H}^{+}+\mathrm{H}(1 \mathrm{~s})$. . Чисельні оцінки отримані для енергій зіткнення 10 і 100 кеВ.

Ключові слова: іон-атомна система із зіткненням, енергетичний підхід

\section{Introduction}

Studying characteristics of the electron, ionatomic collision processes attracts traditionally an intense interest because of the important applications in the astrophysics, plasma and laser physics etc. [1-24]). One of the recent actual problems is investigation of the collisions dynamics between the atoms and surfaces, walls and nanostructures. Modern technological advances have made it possible to perform experiments with full control of the ion-atomic collision systems $[1,2,7]$. Naturally, the collision processes are to be studied for understanding emission spectra of the plasma $[2,10,17,22]$. An important application of the theory of atomic collision theory of plasma is search of the optimum plasma excitation condition for lasing and discovery of new pumping approaches. In addition, these investigations are important to understand the plasma processes themselves. Given systematic data about intensities of spectral lines and respective gins, one can establish basic rules of plasma motion in phase space. The history of plasma spectrum modeling ex- 
tends for decades, starting with the simplest models (see, for instance [2,10]). Different atomic levels are populated in laboratory plasma by different physical processes. This results in a different dependence of each line intensity on the plasma parameters. It had been understood for a long time that this phenomenon could be used for plasma diagnostics. The general principles of such a diagnostic for the simple $\mathrm{H}$-like and He-like ions have been formulated [2]. These diagnostic principles have proven to be useful for understanding the physics of the system and for planning new experiments. In the last years a special interest attracts a studying the electron, ion-atomic collisions in the Debye plasma $[3,6,21]$. Nevertheless, a consistent theory of accounting for effects of the screened Coulomb interaction on the collision dynamics in multielectron atomic systems is absent hitherto. Traditionally a majority of papers is devoted to calculation of characteristics for collision processes in relatively simple systems. In particular, one could mention the proton- $\mathrm{H}$ and hydrogen-like ion, $\mathrm{H}-\mathrm{He}$ collisions, which are accompanying by different impact-excitation, collision ionization and capture phenomena. The usually used theoretical approaches to these problems are the standard quantum-mechanical perturbation theory (PT), the R-matrix approach, the classical trajectory Monte Carlo method etc. [1-6]. For example, in ref. [2] the Monte Carlo method approach has been used to calculate the electron capture and ionization cross sections in hydrogen atom, fully striped ion collisions determined in the Debye-Hückel potential. In ref. [3] the two-centre atomic orbital close-coupling method has been used to calculate the cross-sections of the excitation and electron capture processes in the $\mathrm{H}^{+}+\mathrm{H}(1 \mathrm{~s})$ collision system in a Debye plasma. In the cited paper it has been shown that the dynamics of electron capture and excitation processes is significantly affected by the effect of interaction screening on the direct and exchange electronic couplings and reducing the number of reaction channels. The more information regarding the considered topics can be found in the references [3,6,21]. It is obvious that studying collision dynamics between heavy atoms, multicharged ions and fully striped ions requires a relativistic generalization of the cited methods. Besides, one could mention the cooperative electron, ion-atomic collision processes when the different decay channels (including the production of new particles, the electron-positron pair production (EPPP) in collision of heavy and super heavy ions and nuclei) are opened and must be taken into account simultaneously $[2,11,14,15]$. From this point of view above cited and other methods are dealing with known fundamental theoretical and computational problems $[2,15]$. In our opinion, generally speaking, the problem of adequate treating the ionatomic collision with electron capture and other accompanying processes requires a development consistent quantum electrodynamics (QED) approach or some approximation to QED theory (energy approach). The energy approach (the fundamental aspects) has been developed in refs. [9-14] and then modified and applied to studying a number of problems, namely, studying the electron-ion collision dynamics in the Debye plasma, the resonant states of compound super-heavy nucleus (ions) and EPPP in heavy ions collisions, the recoil induced excitation and ionization in atoms during capture of neutron, the discharge of metastable nuclei during the negative muon capture and collisions of the ultracold atoms with walls and nanostructures etc. [15-23]. In fact it had been applied previously in the study of the purely electronic, electron -nuclear processes in atoms and meso-atomic systems. In this paper the energy approach is generalized to calculate the electron capture cross sections in the $\mathrm{H}^{+}+\mathrm{H}(1 \mathrm{~s})$ collision system. The numerical results are presented for collision energies 10 and $100 \mathrm{keV}$. It is important to note that the presented theory is initially relativistic and, in our opinion, can be used in studying the collision dynamics of heavy atoms, multicharged ions and fully striped ions. Let us recall very interesting task, connected with investigation of the collision dynamics for $\mathrm{H}$-like and $\mathrm{Li}$-like ions $\mathrm{U}^{+91}, \mathrm{U}^{+89}[14]$.

\section{The energy approach to collision problem and model potentials}

In the theory of the non-relativistic atom a convenient field procedure is known for calculating the energy shifts $\Delta E$ of degenerate states because of the interparticle interaction or interaction with an external field $[9,12]$. This procedure is connected with the secular matrix $M$ diagonalization. In constructing $M$, the Gell-Mann and Low adiabatic formula for $\Delta E$ is used. A similar energy approach, using the Gell-Mann and Low formula with the QED scattering matrix, is applicable in the relativistic atom theory [9-14]. The method is a consistently electrodynamics one, allowing for the uniform consideration of a variety of induced and spontaneous 
processes different by their physical nature and with any number of photons. In contrast to the non-relativistic case, the secular matrix elements are already complex in the PT second order (first order of the inter-electron interaction). Their imaginary parts are connected with the radiation decay (collision decay, decay in an external electromagnetic field etc.) possibility. The total energy shift of the state is usually presented in the form [12]:

$$
\Delta E=\operatorname{Re} \Delta E+\mathrm{i} \operatorname{Im} \Delta E \quad \operatorname{Im} \Delta E=-\Gamma / 2,
$$

where $\Gamma$ is interpreted as the level width, and the decay possibility $P=\Gamma$. The whole calculation of the energies and decay probabilities of a non-degenerate excited state is reduced to calculation and diagonalization of the complex matrix $M$. The calculation procedure for $\operatorname{Re} \Delta E$ may be generalised for the case of nearly degenerate states, whose levels form a more or less compact group. One of these variants has been previously used by us $[17,18]$ for a system with a dense energy spectrum, a group of nearly degenerate states is extracted and their matrix $M$ is calculated and diagonalized. If the states are well separated in energy, the matrix $M$ reduces to one term, equal to $\Delta E$. To start with the QED Gell-Mann and Low formula one must choose the zero-order approximation. Usually one uses for this purpose a one-electron Hamiltonian with a central potential that can be treated as a bare potential in the formally exact QED PT. The bare potential includes the electric potential of the atomic nucleus and some model potential of the inter particle interaction. Let us underline that the QED approach is useful in our task as a tool for explaining approximations and a regular method for generalizations in order to take into account additional physical effects. It is obvious that a non-relativistic approximation is quite acceptable in treating the $\mathrm{H}^{+}+\mathrm{H}$ collision dynamics.

In general case let us consider a collision of two similar ions (atoms) with nuclear charge $\mathrm{Z}$ and mass $M$. Following the general formalism we may introduce the bare interaction which can be treated as the zeroth approximation in some formally exact QED PT. As bare potential it is natural to choose non-relativistic electron-nuclear interaction $\mathrm{W}(\mathrm{r}, \mathrm{R})$ and the inter-nuclear interaction $\mathrm{V}($ $R$ ). Here $R$ is distance between the nuclei (ions) and $r$ is the electron coordinate in a centre of the nuclear masses. In a case of the heavy ions (nuclei) collision these potentials include the corresponding terms, connected with account for the finite nuclear size effects and possible strong inter nuclear interaction for small R [14-16]. The smallness parameter of the QED PT is the fine structure constant $\alpha=1 / 137,034$. Under given choice of the bare interaction in the PT zeroth approximation we are dealing with quantum mechanical multi-body problem with known interaction potentials. Such task may be solved within the operator PT $[11,13,17]$, which is called by quantum mechanical PT (QMPT) in a difference from the QED PT [12]. In the QMPT zeroth order a movement of nuclei (nuclear subsystem) is treated independently upon the electron subsystem and described by some equation with potential $V(R)$. One may choose the Dirac equation (DE) for nuclear subsystem in our case only from the point of view of the theoretical consistency and analogy with the electron subsystem, which is described by the DE too. Such an approach is surely more exact than it is necessary for solving the $\mathrm{H}^{+}+$ $\mathrm{H}$ collision problem.

As the potential $W(r, R)$ it is considered the potential of two point Coulomb centres. The full electron-ion interaction can be represented as follows:

$$
W(r, R)=W_{s}(r, R)+\sum_{\lambda} U_{2 \lambda}(r, R) P_{2 \lambda}(r, R),
$$

where a spherically symmetric part is formally as follows: $W_{s}(r, R)=U(R)$, if $r<R$ and $U(r)$, if $r>R$ and $P_{2 \lambda}(r, R)$ are the Legandre polynomials and $U_{2 \lambda}(r, R)$ are the radial parts of the non-spherical interaction. We use here a terminology from the one-centre theories of molecules and quasimolecules (see $[2,14])$. The $U(r)$ can be defined as follows: $U($ $r)=W(r, R=0)$. Naturally in a case of the heavy ions, this potential is defined by a charge distribution in the corresponding nucleus. Surely in a case of the heavy ions, it will contain two continuums (up and down) with boundaries $\pm \tilde{\alpha}^{2}$, where $\tilde{\alpha}=\alpha \cdot Z$ (see $[11,14])$.

In the QMPT zeroth approximation the Hamiltonian of the electron-nuclear system is in a representation of the second quantization as follows:

$$
H_{0}=\sum_{i, j}\left(H_{0 E}\right)_{i j} a_{i}^{+} a_{j}+\sum_{k, l}\left(H_{0 N}\right)_{k l} A_{k}^{+} A_{l},
$$

where $a^{+}, a$ are the electron creation and annihilation operators and $A^{+}, A$ are the same for the nuclear particles (generally speaking quasi-particles corresponding the collective coordinate $R$; see below); $\left(H_{0 E}\right)_{i j}$ and $\left(H_{0 N}\right)_{k l}$ the one-particle matrix elements of the corresponding zeroth-order Hamiltonians for the electron and nuclear subsystems. Formally, the representation for nuclear subsystem 
is given by a spectrum of the DE with some inter nuclear potential $V(R)$ and for the electron subsystem it is given by a spectrum of solutions of the DE with potential of the zeroth approximation $\mathrm{U}_{0}(\mathrm{r})$ $(\mathrm{U}(\mathrm{r}))$. The corresponding system for radial parts of the Dirac function is as follows (in the Coulomb units):

$$
\begin{gathered}
F=-F(æ+|æ|) / T-G\left(E+2 M \tilde{\alpha}^{-2}-U\right) \tilde{\alpha}, \\
G^{\prime}=G(æ-|æ|) / T+F(E-U) \tilde{\alpha},
\end{gathered}
$$

where ж is the Dirac angular quantum number, $E$ is the state energy, $F, G$ being the large and small radial components correspondingly $(F=d F / d T$; $G^{\prime}=d G / d T ;$;); $T$ is the general argument for all system of differential equations (generally speaking for the electron and for the nuclear subsystems). The QMPT perturbation is as follows:

$$
\sum_{i, j}\left(V_{\mathrm{int} E}\right)_{i j} a_{i}^{+} a_{j}+\sum_{i, j, k, l}\left(V_{\mathrm{int} E N}\right)_{i j k l} a_{i}^{+} A_{k}^{+} A_{l} a_{j},
$$

where $\left(V_{i n t E}\right)_{i j}$ is the matrix element of the one-electron operator $U(r)-U_{0}(r),\left(V_{\text {intEN }}\right)_{i j k l}$ is the matrix element of the potential $W_{s}(r, R)-U(r)$.

The differential cross-section for electron capture $n l$ (or ionization $\varepsilon s$ ) in the collision in the lowest QMPT approximation is proportional to the square of the matrix element:

$$
\begin{gathered}
M_{1 s I F \varepsilon s}=\iint d R d r \varphi^{*}(1 s \mid r) \psi^{*}(I \mid R) \times \\
\times\left\{V_{\text {int } E}(r)+V_{\text {int } E N}(r, R)\right\} \psi(F \mid R) \varphi(\varepsilon s \mid r),
\end{gathered}
$$

where $\psi_{I}, \psi_{F}$ are the initial and final state functions for nuclear subsystem (see below). After integration on the electron coordinate the matrix element (5) becomes:

$$
\begin{aligned}
& M_{1 s I F \varepsilon s}=\int d R J_{I F}(R) \Phi_{1 s \varepsilon s}(R), \\
& \Phi_{1 s \varepsilon s}=\int_{0}^{\infty} d r \varphi_{1 s}^{*}\left\{W_{s}(r, R)-U(r)\right\} \varphi_{\varepsilon s}+ \\
& +\int_{0}^{\infty} d r \varphi_{1 s}^{*} \mathrm{H}_{\mathrm{E}} \varphi_{\varepsilon s} .
\end{aligned}
$$

Here $H_{E}$ is the total Hamiltonian with potential $U(R)$. The notation for the production of the nuclear state functions is introduced:

$$
J_{I F}(R)=\psi_{I}^{*}(R) \psi_{F}(R) .
$$

This function includes the total information about the model describing the nuclear subsystem. The function $\Phi_{\text {Ises }}$ includes the total information about the electron subsystem and the model describing the electron-nucleus interaction. Naturally, the last term in (6) is not needed for the present task, as it is formally corresponding to the cooperative processes in a system (EPPP etc.) [11,14]. Following the general receipt of the operator PT [11,13], we suppose that the eigen functions and eigen energies for the potentials $U(r)$ and $U_{0}(r)$ coincide and they are defined by its energy spectrum and the set of the eigen functions without specifying the analytic form of the zero order potential [10]. Such a scheme treats the widely known distorted waves approximation as the zeroth order approximation in the formally exact QMPT allowing for application of the well developed stationary-state methods to the collision problem with variable number of particles and further successive refinement of calculation $[11,13]$. The final electron scattering function $\varphi_{\varepsilon s}$ is constructed as the quadratically integrable eigen function and being the orthogonal complement to set of the discrete state functions in a full analogy with [13]. As it is indicated above, such an approach treats the widely known distorted wave approximation in the zeroth order approximation.

Now we should define the imaginary part of the energy shift provided by a collision process as follows:

$$
\operatorname{Im} \Delta E=-\Gamma / 2=\operatorname{Im} \sum_{n, \varepsilon s, F} \frac{\left(M_{1 s, I, F, \varepsilon s}\right)^{2}}{E_{F}+\varepsilon(n s)-E_{I}-\varepsilon s} .
$$

Here $\Sigma$ means summation over the discrete and integration over the continuum parts of spectra. Indeed, the imaginary contribution is associated with the poles on the energy surface. The individual poles present the concrete channels (capture to bound state, ionization etc.) of reaction with definite final states of the electron (and indeed, nuclear) system. The non-stationary feature of the collision problem manifests itself in the way of the normalization of the nuclear initial and final state functions. Initially the nuclei are free. The final state function $\psi_{F}$ must be normalized to momentum the same as the functions of the virtual states in the formulae (7). The normalization of the nuclear initial state function $\psi_{I}$ is determined by the flow of the initially free nuclei. The value $\Gamma$ given by formula (7) equals to the ionization (capture) cross-section if this function is normalized to unit flow at $\mathrm{R} \rightarrow \infty$. As the zeroth approximation the initial state of the nuclear subsystem is described by the plane wave, which is expanded on the spherical harmonics in order to use the symmetry of task of the zeroth QMPT approximation. In the general case (arbitrary charges of ions, ions with high $\mathrm{Z}$ etc.) surely one should 
introduce the special inter nuclear interaction and exchange the trivial Coulomb potential (as in our task), for example, as it has been done in ref. [22] by introducing the differential equation for the potential $\mathrm{V}(\mathrm{R})$ in the EPPP problem in the heavy nuclei collisions and using the special quantization procedure or in ref. [21,22] by introducing the Yukawa type potential to the electron-ion collision task. This block is used by us in the present paper. In particular, we used the Debye-Hückel potential:

$$
U(r)=-\frac{Z_{a} \cdot Z_{b}}{r} \exp \left(-r / \lambda_{D}\right),
$$

where the Debye screening parameter $\lambda_{D}$ is connected with the plasma parameters such as the temperature $T$ and the charge density $n$ as follows:

$$
\lambda_{D}=\sqrt{k_{B} T / 4 \pi n},
$$

and $k_{B}$ is the Boltzman constant (the electron charge $\mathrm{e}=1$; besides, in our case $\mathrm{Z}=1$ ).

Let us make several important notations. Firstly, naturally in the case of the potential $U(r)$ there are no continuums [11]. Besides, the EPPP channel [14] is not present and naturally the nuclear structure is not detailed (indeed, in our case the nuclei are naturally considered as the point-like charges; $\mathrm{Z}_{\mathrm{a}}=\mathrm{Z}_{\mathrm{b}}=1$ ). The relativistic effects are not important in our case of the $\mathrm{H}^{+}-\mathrm{H}$ collision problem. Nevertheless, we use the relativistic DE as these equations are the basic equations in the corresponding atomic numerical PC code "Superatom" (see description in ref.[8]), which is used by us in this work. Let us note that this code has been earlier used to solve a majority of the atomic and nuclear tasks, including the collision problem of heavy ions [8-24]. At last, the relativistic collision theory is corresponding to our interest to studying the collision dynamics for heavy $\mathrm{H}$-like and $\mathrm{Li}$-like ions such as $\mathrm{U}^{+91}$ and
$\mathrm{U}^{+89}[14]$, where the role of the relativistic effects is obviously very high.

\section{Results and discussion.}

Below we present the results of our studying the electron capture dynamics in the $\mathrm{H}^{+}+\mathrm{H}$ collision system. Calculation has been carried out on the basis of the PC code "Superatom". The calculated cross sections (7) for electron capture to the lowest states at collision energies of $10 \mathrm{keV}$ and $100 \mathrm{keV}$ are presented in tables 1 and 2. The two cases are considered: firstly, the unscreened (pure Coulomb interaction) case and, secondly, for the screened case with the interaction screening parameter $\lambda_{D}=4 a_{0}$ ( $a_{0}$ is the Bohr radius). From physical point of view in the second case one may deal with quite dense plasma (the thermonuclear reactor plasma etc.). In table 1 we compare the our data on the electron capture (state $1 s$ ) cross sections for unscreened and screened $\left(\lambda_{D}=4 a_{0}\right)$ cases with the analogous data from ref. [3]. In table 2 we present our data on cross-sections of the electron capture (state $\mathrm{nl}$ : $n=2,3 ; l=0,1,2)$ cross sections for the unscreened and screened $\left(\lambda_{D}=4 a_{0}\right)$ cases for the same collision energies $E=10$ and $E=100 \mathrm{keV}$.

Table 1

Comparison of calculated electron capture (state $1 \mathrm{~s}$ ) cross sections (in units of $10^{-16} \mathrm{~cm}^{2}$ ) for unscreened and $\lambda_{D}=4 a_{0}$ screened cases at $E=10$ and $E=100 \mathrm{keV}$ with similar data from [3].

\begin{tabular}{|c|c|c|}
\hline$E(\mathrm{keV})$ & $1 s[3]$ & $1 s$ \\
\hline No screening & 7.87057 & 7.87142 \\
10 & 0.08354 & 0.08431 \\
\hline 100 & 7.31461 & 7.31627 \\
10 & 0.06394 & 0.06504 \\
\hline
\end{tabular}

Comparison of calculated electron capture (state $n l: n=2,3 ; l=0,1,2)$ cross sections (in units of $10^{-16} \mathrm{~cm}^{2}$ ) for unscreened and $\lambda_{D}=4 a_{0}$ screened cases at $E=10$ and $E=100 \mathrm{keV}$

\begin{tabular}{|c|c|c|c|c|c|}
\hline$E(k e V)$ & $2 s$ & $2 p$ & $3 s$ & $3 p$ & $3 d$ \\
\hline No screening & 0.20998 & 0.35312 & 0.01908 & 0.04621 & 0.02615 \\
10 & 0.01882 & 0.00422 & 0.00684 & 0.00233 & 0.00064 \\
100 & 0.10899 & - & - & - & - \\
10 & 0.00316 & - & - & - & - \\
\hline With screening & & & & - \\
\hline
\end{tabular}

In a whole, our results on the cross-sections of capture to the 1s state for the unscreened case and the case with screening are in a good agreement with the similar data from ref. [3]. A little difference between the presented results is probably explained by using the different approaches in the present 
paper and ref. [3]. In fact, it is connected with using the different atomic orbital basis's and different formula for the corresponding cross-section. In any case, we can conclude that the presented energy approach is successfully tested for the $\mathrm{H}^{+}+\mathrm{H}(1 \mathrm{~s})$ collision system in the Debye plasma. We believe that our approach can be effectively used for studying the electron capture processes in the more complicated ion-atomic collision systems, where an application of other standard theoretical approaches [2-7] can deal with the serious fundamental and numerical problems (see discussion in ref. [2]).

Acknowledgement. The useful critical comments of the anonymous referees are very much acknowledged.

\section{Reference}

1. Henkel C., Schmiedmayer J., Westbrook C. (Eds.), Special Issue - Atom chips: manipulating atoms and molecules with microfabricated structures//Europ. Phys.J.D. - 2005. - Vol.35, N1.

2. Photonic, Electronic and Atomic Collisions. Eds. F.Aumar, H.Winter, World Sci., Singapore. 2007. - P.1-650.

3. Zeng S L, Liu L., Wang J.G., Janev R.K., Atomic collisions with screened Coulomb interactions: excitation and electron capture in $\mathrm{H}++\mathrm{H}$ collisions// J. Phys. B: At. Mol. Opt. Phys. - 2008. - Vol.41. P.135202

4. Arnecke F., Friedrich H., Madrofinero A., Quantum effects in collisions of ultracold atoms with walls and nanostructures//Journ. Phys. CS. - 2007. Vol.88. - P.012041-1--012041-6.

5. Friedberg R., Hartmann S. R., Manassah J. T., Frequency shifts in emission and absorption by resonant systems of two-level atoms// Phys. Rep.2003. Vol.37. - P.101-179.

6. Okutsu H., SakoI., Yamanouchi K., Diercksen G.H.F., Electronic structure of atoms in laser plasmas: a Debye shielding approach// J.Phys.B. - 2005. Vol.38. - P.917-927.

7. Nakamura N., Kavanagh A.P., Watanabe H., Sakaue H.A., Li Y., Kato D., Currell F.J., Ohtani S., Relativistic effects on resonant interactions between electrons and highly charged ions// J.Phys.CS. - 2007. Vol.88. - P.012066-1--10.

8. Glushkov A.V., Atom in electromagnetic field. - Kiev: KNT, 2005. - 400P.

9. Ivanova E.P., Ivanov L.N., Glushkov A.V., Kramida A.E., High order corrections in relativistic perturbation theory with model zeroth approximation// Phys. Scripta. - 1985. - V.32,N4. - P.512-524.

10. Ivanov L.N., Ivanova E.P., Knight L.V., Glushkov A.V. Spectrum of plasma containing $\mathrm{Ne}$-and $\mathrm{Na}$-like ions:
Consistent account for Rydberg and autoionization Rydberg series in balance equations// Phys. Scripta. -1996 . - Vol.53. - P.653-667.

11. Glushkov A.V., Ivanov L.N., Electron-positron pair production in heavy atomic nucleus collisions: New consistent quantum-mechanical approach//Preprint of the Institute for Spectroscopy of the USSR Academy of Sciences. - 1991. - NAS-1. - 15P.

12. Glushkov A.V., Ivanov L.N., Radiation Decay of Atomic States: atomic residue and gauge non-invariant contributions // Phys. Lett.A. - 1992. Vol.170,N1. - P.33-38.

13. Glushkov A.V., Ivanov L.N., DC strong-field Stark effect: consistent quantum-mechanical approach// J. Phys. B: At. Mol. Opt. Phys. - 1993. - Vol.26. P.L379-L388.

14. Glushkov A.V., Rusov V.D., Ambrosov S.V., Loboda $A . V$. Resonance states of compound super-heavy nucleus and EPPP in heavy nucleus collisions // In: New projects and new lines of research in nuclear physics.Eds. G.Fazio, F.Hanappe, Singapore : World Scientific. - 2003. - P.126-182.

15. Glushkov A.V., Malinovskaya S.V., Dubrovskaya Yu.V., Vitavetskaya L.A., Quantum calculation of cooperative muon-nuclear processes: discharge of metastable nuclei during negative muon capture// Recent Advances in Theory of Phys. and Chem. Systems (Springer). - 2006. - Vol.15. - P.301-308.

16. Glushkov A.V., Gurnitskaya E.P., Loboda A.V., Advanced quantum mechanical calculation of superheavy ions: energy levels, Radiation and Finite Nuclear size effects// Low Energy Antiproton Phys. (AIP). - 2005. - Vol.796. - P.217-220.

17. Glushkov A.V., Loboda A.V., Ambrosov S.V., Gurnitskaya E.P., Prepelitsa G.P., Consistent QED approach to calculation of electron-collision excitation crosssections and strengths: Ne-like ions // Int. Journ. Quant.Chem. - 2005. - Vol.104, N4 . - P. 562569.

18. Glushkov A.V., Loboda A.V., Malinovskaya S.V., Gurnitskaya E.P., Korchevsky D.A., Diagnostics of the collisionally pumped plasma and search of the optimal plasma parameters of $\mathrm{x}$-ray lasing: Calculation of electron-collision strengths and rate coefficients for Ne-like plasma// J.Phys.CS. - 2005. - Vol.11. P.188-198

19. Glushkov A.V., Malinovskaya S.V., Gurnitskaya E.P., Khetselius O.Yu.,Dubrovskaya Yu.V., Consistent quantum theory of the recoil induced excitation and ionization in atoms during capture of neutron// J.Phys.CS. - 2006. - Vol.35. - P.425-430

20. Glushkov A.V., Khetselius O.Yu., Loboda A.V., Svinarenko A.A., QED approach to atoms in a laser field: Multi-photon resonances and above threshold ionization//Frontiers in Quantum Systems in Chemistry and Physics (Springer). - 2008. - Vol.18. - P.541558. 
21. Glushkov A.V., Loboda A.V., Khetselius O.Yu., Gurnitskaya E.P., Korchevsky D.A., Prepelitsa G.P., Sensing the electron-collision excitation cross-sections for $\mathrm{Ne}$-like ions of Fe in a plasma in the Debye shielding approximation// Sensor Electr. and Microsyst. Techn. - 2007. - N2. - P.9-13

22. Loboda A.V., Gurnitskaya E.P., Korchevsky D.A., Sensing the optimal plasma parameters for X-ray lasing: calculation of electron-collision excitation cross- sections for Ar-like plasma ions//Sensor Electr. and Microsyst. Techn. - 2006. - N1. - P.18-22.

23. Loboda A.V., Elementary processes in a collisionally pumped plasma: Calculation of the electron-collisional excitation cross-sections in the Ne-like plasma// Phys. Aerodisp.Systems. - 2006. - Vol.41. - P.139-144.

24. Loboda A.V., Quantum theory of collisions of the ultracold atoms with walls and nanostructures// Photoelectronics. - 2008. - N17. - P.71-74. 